\title{
Etudiants et enseignants face aux travaux pratiques de physique en 1ère année de DEUG
}

C. Génin et A. Pellet

\section{Q OpenEdition}

12 Journals

Édition électronique

URL : http://journals.openedition.org/trema/2349

DOI : 10.4000/trema.2349

ISSN : 2107-0997

\section{Éditeur}

Faculté d'Éducation de l'université de Montpellier

\section{Édition imprimée}

Date de publication : 1 mai 1993

Pagination : 93-107

ISSN : 1167-315X

\section{Référence électronique}

C. Génin et A. Pellet, « Etudiants et enseignants face aux travaux pratiques de physique en 1ère année de DEUG », Tréma [En ligne], 3-4 | 1993, mis en ligne le 01 mai 1993, consulté le 06 mai 2019. URL : http://journals.openedition.org/trema/2349; DOI : 10.4000/trema.2349

Ce document a été généré automatiquement le 6 mai 2019.

Trema 


\title{
Etudiants et enseignants face aux travaux pratiques de physique en 1ère année de DEUG
}

\author{
C. Génin et A. Pellet
}

1 Le rapport à l'expérimental dans l'enseignement des sciences, et particulièrement de la Physique, est classiquement paré de toutes les vertus. Sur le mode lyrique, opposant le « concret » au « formel », ou sur celui d'une étude plus précise des objectifs qu'on peut lui attribuer, beaucoup s'accordent sur l'énoncé suivant : les difficultés de l'enseignement de la Physique proviennent de ce qu'il n'est pas basé sur des manipulations pratiques.

2 Ce discours repose souvent sur des hypothèses implicites très fortes quant au fonctionnement de l'élaboration scientifique (qui procéderait uniquement d'une observation rigoureuse des faits) et aux mécanismes de l'apprentissage (qui seraient aussi principalement de caractère empirique et inductif). Il est, de plus, souvent supposé que ces deux fonctionnements peuvent à tout moment être mis en correspondance terme à terme. Si cela était, les difficultés rencontrées dans l'enseignement des sciences ne pourraient être dues qu'à des causes exogènes : manque de moyens, manque de formation pédagogique notamment.

3 Ce véritable postulat pédagogique est ancré de longue date dans le corps enseignant et est encore largement majoritaire aujourd'hui. Les études précises qui viendraient étayer ces a priori sont en fait assez rares. Quand de telles études existent, les conclusions en sont parfois étonnantes.

4 Ainsi, Yager, Englen et Snider (1969) ont comparé trois groupes d'élèves (13-14 ans) étudiant la Biologie.

- Dans le groupe « Discussion », les élèves reçoivent une description des 50 expériences du cursus et en étudient les résultats. Mais ils ne les voient, ni ne les pratiquent jamais euxmêmes.

- Dans le groupe « Démonstration », les expériences sont montrées mais pratiquées par le professeur.

- Dans le groupe « Laboratoire ", les élèves pratiquent toutes les expériences eux-mêmes. 
Les auteurs ne constatent aucune supériorité du troisième groupe dans aucun domaine, excepté les tâches manipulatrices. Des résultats du même type ont été présentés par d'autres auteurs (Coulter, 1966). Glasson (1989) est à peine plus optimiste, il mesure une supériorité de l'approche au laboratoire, mais uniquement en ce qui concerne certains savoirs "procéduraux», mal définis au demeurant. Herron (1971) montre ainsi que la grande majorité des activités pratiquées est fortement « guidée ». Le problème à traiter, les moyens de le traiter, les réponses à vérifier, tout est donné aux élèves, ce qui produit un fort degré de directivité.

6 De nombreux auteurs ont cherché à étudier différents aspects de l'activité expérimentale. Ils sont assez divers. Sans se livrer à une typologie complète, on peut au moins citer quelques grandes catégories :

- Une réflexion sur des expériences qui seraient à la fois plus simples et plus convaincantes que celles couramment pratiquées (Read, 1987). Ceci fournit une très large littérature, typique des publications propres aux enseignants, sans que l'on perçoive toujours clairement en quoi la structure profonde des Travaux Pratiques (T.P.) traditionnels en serait modifiée.

- Des études de mise en pratique de modèles simples dans des cas d'application complexe. L'accent y est souvent mis sur les techniques d'approximation, les intervalles de confiance, etc.. L'assistance par ordinateur y est assez fréquente (Durey. Laurent, Journeaux, 1983 ; Durey, Journeaux, 1987 ; Firpo. Neel et Boyer, 1989 ; Rosell, Valero, Llopis, 1983 ; Herrera, Corullon, 1987). L'insistance est mise ici sur l'aspect réaliste des difficultés manipulatoires et de leur traitement.

- Plus rarement, on peut trouver dans la littérature des travaux visant à un apprentissage spécifique de modes d'analyse expérimentaux : l'expression graphique en particulier. Là encore, l'assistance par ordinateur est un fait courant (Mokros 1987 ; Nachmias et Linn, 1987).

- Enfin, des travaux de plus en plus nombreux intègrent des phases d'expérimentation dans des processus, plus ou moins guidés, d'élaboration et d'acquisition de modèles (Perez et Rubio, 1986 ; Gutierrez et Rodriguez, 1987 ; Johsua et Dupin, 1989).

7 On peut donc constater à quel point sont diverses les approches expérimentales, surtout parmi les critiques les plus convaincus des activités traditionnelles. Ceci devrait peut-être nous amener à un retour sur ces critiques elles-mêmes; sans doute sont-elles trop hâtives et ne peuvent-elles prendre leur véritable sens, éventuellement, qu'après une analyse plus profonde des fonctions didactiques du recours à l'expérimental ? (Johsua et Johsua, 1987 ; Johsua et Johsua, 1988). L'idée de base, pour ce faire, est celle d'une nécessaire transposition didactique (Chevallard, 1985) entre le fonctionnement savant et la pratique d'enseignement. Dans l'enseignement expérimental, il apparait toujours une coupure entre l'expérience et le modèle. En général, on se convainc de la validité du modèle par son application pratique (validation opératoire). Pour l'essentiel, les T.P. de l'enseignement supérieur français relèvent de la validation opératoire.

On peut aisément montrer qu'il est naïf de croire que cette façon de procéder facilite, quant au fond, l'acquisition du modèle (cf. ci-dessus les résultats de travaux de recherche qui contredisent cet espoir). Cela ne signifie pas que certaines fonctions didactiques ne sont pas remplies par cette approche, plus prosaïques peut-être, mais bien réelles celleslà. Ce sont :

- des apprentissages spécifiques, liés soit aux appareils de mesure et à la métrologie en général, soit à des techniques manipulatoires spécifiques (séquences d'une analyse chimique 
par exemple), soit à une combinaison de ces deux aspects. Ces apprentissages ne peuvent être abordés qu'en situation de manipulation pratique ; c'est la conclusion claire des recherches citées ci-dessus en la matière.

- des apprentissages de certains outils conceptuels. Ce point est le plus délicat, parce que le plus mal connu et étudié à la fois. Il s'agit d'apprendre à présenter un compte rendu d'expérience, à dominer les différents aspects de l'outil graphique, à évaluer la pertinence de mesures, etc... Ce sont des apprentissages qui se traitent essentiellement en T.P., dans le cadre traditionnel de la « validation opératoire ». Il s'agit là d'une constatation qui demanderait à être elle-même analysée : ces apprentissages sont-ils intrinsèquement propres à l'approche expérimentale, ou sont-ils classés dans ce cadre seulement à la suite d'une tradition particulière d'enseignement?

\section{Présentation de l'étude}

9 L'étude présentée ici est une étude préliminaire. L'objectif général futur est d'élucider les fonctions didactiques réelles des T.P. de Physique à l'Université, et de décrire les alternatives existantes, le coût didactique de leur mise en pratique, ainsi que d'estimer leur viabilité. Dans l'immédiat, avant d'analyser le contenu et le déroulement des T.P. traditionnels dans le détail, nous avons cherché à éclaircir comment leurs fonctions apparaissaient, subjectivement, aux acteurs : étudiants et enseignants.

\section{Méthode}

Après Ben-Zvi (Ben-Zvi, Hostein et Samuel, 1977) et Sebastia (1987), nous avons proposé un questionnaire demandant leur appréciation sur les T.P. de Physique à des étudiants de première année d'université scientifique et à leurs enseignants (Université de Provence). L'échantillon est constitué de la manière suivante :

- 291 étudiants de Sciences et Structure de la Matière (Mathématiques et Physique) (SSM).

- 211 étudiants de Sciences de la Nature et de la Vie (Chimie et Biologie) (SNV).

- 14 enseignants (ENS) de l'unité de module Enseignement expérimental de $1^{\circ}$ année.

11 Le questionnaire a été proposé à la fin de l'enseignement expérimental. Les sujets doivent donner leur estimation sur des énoncés qui sont susceptibles de caractériser les T.P. à l'aide d'une échelle de 1 à 4 (tout à fait en désaccord, plutôt en désaccord, plutôt d'accord, tout à fait d'accord) (Ghiglione et Matalon, 1978). Les énoncés sont traités deux fois : la première concerne le bilan tiré des T.P. réellement pratiqués, la deuxième ce que devraient, dans l'idéal, être des travaux pratiques dans un cursus universitaire.

Les énoncés proposés peuvent, à priori, être regroupés dans des catégories d'objectifs d'apprentissage particulières (cf annexe 1). Elles sont au nombre de quatre :

- «Illustration du cours » noté « I » (Prop.l, 5, 6, 14, 16, 18, 19, 21)

- «Techniques manipulatoires » noté « $T$ » (Prop.2, 3, 8, 10, 13, 15, 20, 23)

- «Attitudes scientifiques » noté « AS » (Prop.4, 7, 12, 17, 25, 26)

- «Attitudes générales » noté «AG » (Prop.9, 11, 22, 24, 26).

Il sera intéressant, lors du traitement des résultats, de vérifier si ces regroupements a priori ont une pertinence pour l'interprétation des réponses.

D'une manière générale, l'analyse des réponses débutera par l'étude de la signification des fréquences brutes de réponse, en fonction du groupe (SSM, SNV ou Enseignants), pour 
la partie « réalité » et la partie «idéal ». Ensuite une comparaison réalité / idéal sera effectuée pour chaque groupe.

\section{Résultats}

\section{Etudes des fréquences brutes des résultats}

Le tableau donné dans l'annexe 1 indique, pour chacune des 26 propositions, les pourcentages des répondants dans chacune des modalités 1, 2, 3, 4 concernant la « réalité » (A) d'une part, et l' «idéal » (B) d'autre part (les pourcentages sont effectués sur l'ensemble des réponses valides). Ce tableau montre de façon évidente qu'une grande partie des 26 propositions caractérise les T.P. existants. Au niveau des souhaits, l'accord avec les propositions est encore plus net : aucune d'entre elles n'est rejetée par plus d'un tiers des répondants.

Dans ce qui suit, bien que nous soyons conscients du peu de crédit à accorder aux convictions des réponses 2 et 3 (répondants indécis), nous avons pour des raisons évidentes de simplification, regroupé d'une part les modalités 1 et 2 signifiant le désaccord, d'autre part les modalités 3 et 4 exprimant l'accord. De plus nous nous sommes intéressés aux pourcentages de réponses dans chacun des groupes SSM, SNV et Enseignants. Bien que le nombre de répondants de ce dernier groupe soit faible (14) et que les pourcentages ne soient pas très significatifs, nous avons tenu à présenter les résultats correspondants afin de pouvoir les comparer à ceux des autres groupes puisque ce sont ces enseignants qui gèrent le fonctionnement des T.P.

\section{Propositions pour lesquelles l'accord est le plus important}

\section{A. Dans la réalité, ou « ce que sont les T.P."}

Pour chaque groupe, nous avons classé par ordre décroissant les propositions avec lesquelles plus de $60 \%$ de répondants sont en accord, en caractérisant chacune d'elles par la catégorie d'objectifs d'apprentissage à laquelle elle appartient. En observant le tableau (annexe 2), il apparait de façon évidente une prédominance des catégories " $T$ " (développement des techniques) et «I» (illustration du cours). De façon plus précise, on obtient pour chaque groupe :

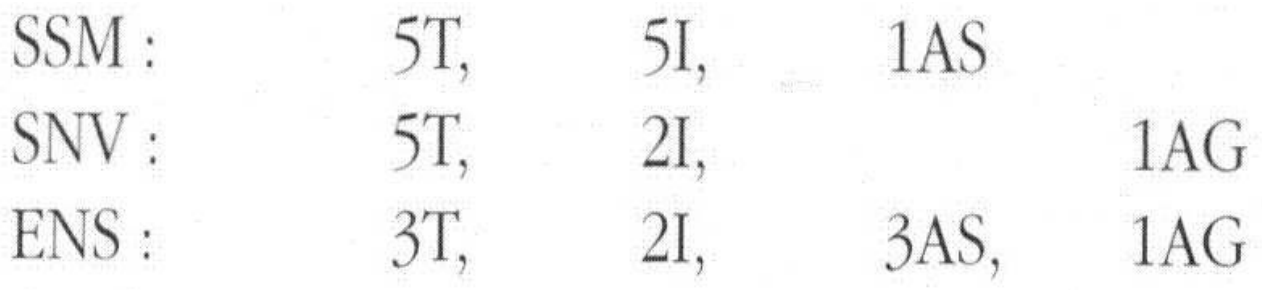

\section{Ecarts entre les groupes}

- Ecarts SSM-SNV : La différence entre les deux groupes d'étudiants est essentiellement due à un accord avec les propositions plus massif pour le groupe SSM que pour le groupe SNV. Les propositions pour lesquelles l'écart est le plus important sont : 


\section{P8 (I) (Concevoir et réaliser une expérience) 70\%SSM, 49\% SNV. \\ P4 (AS) (Interpréter résultats) \\ P14 (I) (Appliquer résultats) \\ $70 \%$ SSM, $\quad 50 \%$ SNV. \\ 66\% SSM, $\quad 48 \%$ SNV.}

19 Au sujet de P8, il sera bon de vérifier, dans un travail ultérieur, de la façon la plus objective possible, si cette proposition est effectivement mise en œuvre dans les T.P. existants. Cette mise en œuvre ne semble être effectuée que pour $29 \%$ des enseignants. Il apparaît alors que les étudiants SNV sont plus lucides que ceux du groupe SSM. Quant à l'interprétation des résultats $(\mathrm{P} 4)$ l'écart entre les groupes semble être dû à une difficulté plus grande des étudiants de SNV pour interpréter la Physique. Il paraît bien évident aussi que les étudiants de SNV ne sont pas majoritairement d'accord avec la proposition P14 car en fait ils établissent peu de résultats théoriques. résultats se regroupent par catégorie de la façon suivante :

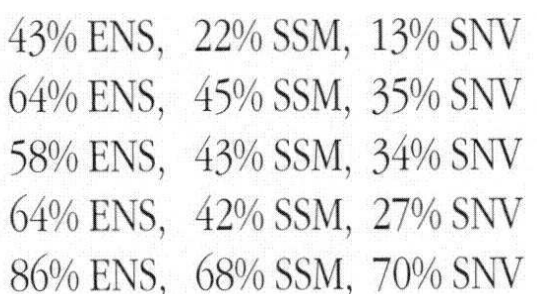

P13 (T) (Présenter résultats)

P7 (AS) (Apprécier ordre grandeurs)

P11 (AG) (Relations avec enseignants)

P12 (AS) (Esprit critique)

Ces propositions obtiennent un accord massif des enseignants sur ce que devraient être les T.P. Il est alors raisonnable de supposer qu'il leur est difficile de trouver que ces objectifs ne sont pas réalisés alors qu'ils mettent tout en œuvre pour qu'il en soit ainsi. Inversement les propositions pour lesquelles l'accord des étudiants est plus important que celui des enseignants sont :

\section{P8 (T) (Concevoir et réaliser expérience) 70\% SSM, 49\% SNV, 29\% ENS. $\mathrm{P} 10$ ( T ) (Suivre instructions) $\quad 72 \% \mathrm{SNV}, 60 \% \mathrm{SSM}, 36 \% \mathrm{ENS}$. P15(T) (Observer et recueillir informations) 73\% SSM, 63\% SNV, 50\% ENS.}

Une analyse précise des feuilles de manipulations distribuées aux étudiants ainsi que des observations sur le comportement des étudiants au cours des séances de T.P. permettront d'analyser ces différences. Toutefois notre connaissance du fonctionnement des T.P. permet de dire que les étudiants sont souvent amenés à suivre des instructions précises plutôt que de concevoir une expérience. Une fois de plus, les étudiants du groupe SNV paraissent plus proches de la réalité.

\section{B. Dans les souhaits,ou «ce que devraient être les T.P.»}

Nous avons classé, pour chaque groupe, par ordre d'accord décroissant, les propositions avec lesquelles au moins $90 \%$ des répondants sont d'accord (voir tableau annexe 2). Ces 


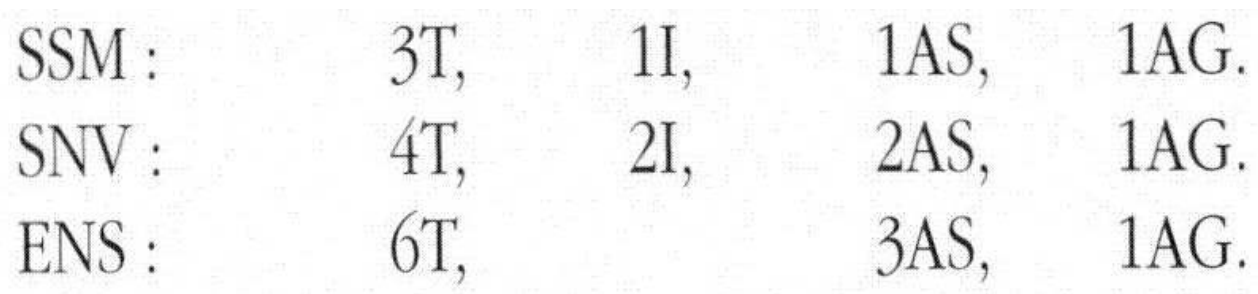

Ici aussi les propositions des catégories « $\mathrm{T}$ » et « $\mathrm{I}$ » sont prédominantes.

\section{Ecarts entre les groupes}

- Ecarts SSM-SNV : On remarque que les étudiants de SNV sont plus « demandeurs » que les étudiants de SSM : 9 propositions sont souhaitées par au moins $90 \%$ des étudiants SNV alors que l'on ne compte que 6 propositions dans les mêmes conditions pour le groupe SSM. Toutefois les écarts pour une proposition donnée sont toujours inférieurs à $10 \%$.

- Ecarts Enseignants-Etudiants : Les propositions souhaitées par les enseignants le sont très fortement ( 8 propositions obtiennent $100 \%)$. On remarque des écarts importants entre les groupes Enseignants et les groupes Etudiants. Les enseignants accordent beaucoup plus d'importance que les étudiants à l'appréciation des ordres de grandeur et à la rédaction d'un compte rendu :

\section{P7 (AS) (Apprécier ordre de grandeur) $\quad$ 100\% ENS, 76\% SNV, 75\% SSM. P3 (T) (Faire compte rendu ) 93\% ENS, 70\% SNV, 70\% SSM.}

Par contre, les exigences des étudiants sont plus importantes que celles des enseignants pour les propositions $1,6,10,21$ et 25 , que l'on peut regrouper de la façon suivante :

\section{P21 (I) (Mémoriser les formules) \\ $73 \%$ SNV, 64\% SSM, 8\% ENS. P6 (I) (Vérifier théorie) 87\% SSM, $86 \%$ SNV, $\quad 64 \%$ ENS. P1 (I) (Comprendre concepts) 92\% SSM, $\quad 92 \%$ SNV, $\quad 71 \%$ ENS.}

29 Les différences obtenues pour ces propositions se justifient par l'hypothèse que les étudiants aimeraient bien que les T.P. les aident à améliorer leurs performances dans les U.M. théoriques. 67\%SSM et 33\% ENS, portent à penser que les étudiants aussi bien SSM que SNV, peu habitués au lycée à avoir des initiatives au niveau des manipulations, sont nombreux à avoir besoin de se sentir sécurisés. Par contre les enseignants aimeraient plus d'initiative de la part des étudiants.

ENS, les pourcentages obtenus montrent un réalisme plus important chez les enseignants que chez les étudiants.

On peut remarquer aussi que les enseignants ne font apparaître de façon majoritaire, dans leurs souhaits, aucune proposition qui relève de l'illustration du cours. Traditionnellement ce rôle était joué, non pas par les T.P., mais par les expériences de cours. Or celles-ci ont complètement disparu (manque de matériel, de personnel technique mais aussi de temps Enseignant-Etudiant). Par contre les enseignants mettent beaucoup plus l'accent sur les objectifs d'attitude scientifique. 


\section{Propositions pour lesquelles le désaccord est le plus important} il s'agit de P26 (Temps nécessaire), de P9 (Travailler en équipe) et de P12 (Esprit critique). Il serait intéressant de savoir s'il s'agit dans les trois cas des mêmes étudiants, et de s'interroger sur la signification de ce rejet.

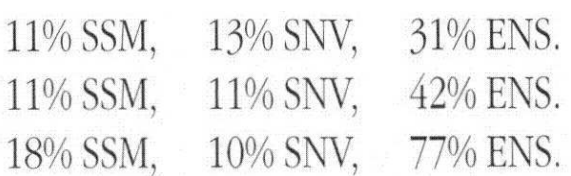

$37 \%$ SSM, $59 \%$ SNV, $\quad 43 \%$ ENS. $37 \%$ SSM, $\quad 45 \%$ SNV, $\quad 57 \%$ ENS. $37 \%$ SSM, $40 \%$ SNV, $64 \%$ ENS. 32\% SSM, 39\% SNV, 54\% ENS. mbre de propositions et avec des pourcentages sont souhaitées ou pas par l'un ou l'autre des trois groupes :

- La proposition P21 n'est souhaitée que par $8 \%$ des enseignants, mais par $64 \%$ de SSM et $73 \%$ de SNV. Le fait qu'elle soit faiblement souhaitée par les enseignants explique sa non réalisation.

- La proposition P3 est souhaitée par l'ensemble des trois groupes (70 \% SSM,70 \% SNV, 93 \% ENS), mais le temps nécessaire à la correction des comptes rendus rend sa réalisation difficile.

Il en est de même pour les propositions P20 (souhaitée par $90 \%$ de SSM, $89 \%$ de SNV et $79 \%$ d'ENS) et P25 (79\% de SSM, $94 \%$ de SNV et $62 \%$ d'ENS). Les difficultés de mise en œuvre : coût financier des appareillages, difficulté d'introduire des groupes d'étudiants dans les laboratoires de recherche sont probablement responsables de ces écarts réalité/ souhaits. On peut noter pour P20 et P25 que les souhaits des enseignants sont moins massifs que ceux des étudiants, ceci étant dû à un sens des réalités plus important.

\section{B. Dans les souhaits,ou " ce que ne devraient pas être les T.P."} de répondants assez élevés. Les propositions rejetées par les trois groupes sont:

$\begin{array}{llll}\text { P18 (I) (Etre lié au cours ) } & 11 \% \text { SSM, } & 13 \% \text { SNV }, & 31 \% \text { ENS. } \\ \text { P10 (I) (Suivre instructions) } & 11 \% \text { SSM, } & 11 \% \text { SNV, } & 42 \% \text { ENS. } \\ \text { P21 (I) (Mémoriser formules) } & 18 \% \text { SSM }, & 10 \% \text { SNV }, & 77 \% \text { ENS. }\end{array}$

Le groupe SSM fait apparaître un rejet non négligeable (entre 10 et $13 \%$ ) de trois propositions que l'on ne retrouve pas dans les autres groupes et qui est très surprenant : 
Le groupe des enseignants est plus ou moins violemment contre un plus grand nombre de propositions. Celles qui n'apparaissent pas chez les étudiants sont de plusieurs types :

- P11 (Relations avec enseignants : 17 \%) et P26 (Temps nécessaire : $14 \%$ ) ne correspondent probablement pas à une conception idéologique d'une minorité d'enseignants.

- Le rejet de P16 (Enseigner à partir d'expériences : 17 \%) correspond à la volonté de quelques enseignants de ne pas concevoir les relations théorie/ expérience comme expérience au départ et modèle en conclusion (option du collège et du lycée) ; par contre le rejet de P19 (Illustrer une théorie : $15 \%$ ) et de P6 (Vérifier une théorie : $14 \%$ ) exprime l'opinion de certains enseignants qui refusent de considérer les T.P. comme une vérification expérimentale des axiomes. Il serait peut-être utile que les enseignants de T.P. confrontent leurs opinions sur ces deux options.

\section{Croisement entre les réponses réalité/souhaits : satisfaction- mécontentement}

Nous avons voulu tester si, dans l'ensemble, les questionnés paraissaient satisfaits du fonctionnement des T.P, à l'Université de Provence. Pour chaque proposition énoncée et pour chaque répondant, on a réalisé un croisement entre sa réponse dans la colonne $\mathrm{B}$ (qui reflète son attente) et sa réponse dans la colonne A (qui correspond à ce qu'il perçoit de la réalité). Pour chaque proposition le tableau suivant est obtenu.

Réponse Col. B
2

3

\section{Réponse}

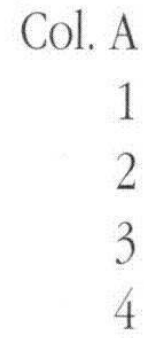

$\begin{array}{lll}1 & \text { A1B1 } & \text { A1B2 } \\ 2 & \text { A2B1 } & \text { A2B2 } \\ 3 & \text { A3B1 } & \text { A3B2 } \\ 4 & \text { A4B1 } & \text { A4B2 }\end{array}$

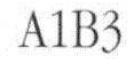

A1B4 
entre les deux. La moyenne de l'indice de satisfaction a été calculée sur l'ensemble des 26 propositions pour chacun des groupes; on obtient : $\mathrm{Is}_{\mathrm{SSM}}=64 ; \mathrm{Is}_{\mathrm{ENS}}=63 ; \mathrm{Ls}_{\mathrm{SNV}}=55$. Il nous a paru intéressant de connaître la satisfaction des usagers des T.P. seulement pour ce qui existe. On se contentera alors d'une cohérence moins forte entre souhaits et réalité et le nouvel indice de satisfaction sera: Is+ $=\left(\mathrm{A}_{3} \mathrm{~B}_{3}+\mathrm{A}_{4} \mathrm{~B}_{4}+\mathrm{A}_{3} \mathrm{~B}_{4}+\mathrm{A}_{4} \mathrm{~B}_{3}\right) \times 100 / \mathrm{Nbre}$ de répondants)

Si l'on regarde plus en détail les résultats obtenus pour chaque proposition, on note que les SSM sont plus satisfaits que les SNV pour 23 propositions et la valeur moyenne des écarts entre indices est de l'ordre de 11. Les propositions qui obtiennent le plus fort indice Is+ communes aux deux groupes d'étudiants SSM et SNV sont :

$\begin{array}{lll}\text { P2 (Utiliser appareils de mesure) (T) } & 86 & 80 \\ \text { P6 (Vérifier une théorie) (I) } & 72 & 59 \\ \text { P23 (Méthodes générales d'expérience) (T) } & 69 & 64 \\ \text { P15 (Observer et recueillir informations) (T) } & 68 & 53 \\ \text { P5 (Physique et phénomènes concrets) (I) } & 64 & 59 \\ \text { P1 (Aider à comprendre la physique) (I) } & 62 & 49\end{array}$

Seules les catégories « $T$ » et «I» sont présentes. Pour le groupe des Enseignants les propositions obtenant le plus grand indice de satisfaction sont :

P13 (Présenter résultats) (T) 88

P1 (Aider à comprendre la physique) (I) 77

P23 (Méthodes générales d'expériences) (T) 77

P2 (Utiliser appareils) (T) 66

P4 (Interpréter résultats) (AS) 66

P9 (Travailler en équipe) (AG) 66

47 Comparer Is ${ }_{\text {ENS }}$ avec Is ${ }_{\text {ETU }}$ c'est dans une certaine mesure voir si le "message " que pensent avoir fait passer les enseignants est effectivement bien passé auprès des étudiants. Cela semble donc réalisé pour les propositions suivantes communes aux trois groupes. Ce sont: P2 (Utiliser appareils), P23 (Méthodes générales d'expériences), P15 (Observer et recueillir informations) et P1 (Aider à comprendre la physique).

On remarque que pour 5 propositions la différence entre les indices SSM et SNV est relativement importante, de l'ordre de 20 . Il s'agit de :

P4 (Interpréter les résultats) 70 pour SSM et 51 pour SNV,

P8 (Concevoir et réaliser une expérience) 66 pour SSM et 48 pour SNV,

P12 (Esprit critique) 60 pour SSM et 33 pour SNV,

P13 (Présenter résultats) 66 pour SSM et 46 pour SNV, 
P14 (Appliquer résultats) 72 pour SSM et 58 pour SNV.

Les étudiants de SNV paraissent plus « bloqués » que ceux de SSM lors de l'interprétation et l'application des résultats; il leur manque en effet la plupart du temps des résultats théoriques auxquels ils pourraient se référer.

\section{Mécontentement ou déception des utilisateurs des T.P.}

Dans cette partie il nous a paru plus significatif pour parler de mécontentement de ne tenir compte que des étudiants qui sont tout à fait d'accord sur le fait que la proposition doit caractériser les T.P. et qui trouvent qu'elle ne les caractérise pas dans la réalité. L'indice de mécontentement est alors défini par: $\operatorname{Im}=\left(\mathrm{A}_{1} \mathrm{~B}_{4}+\mathrm{A}_{2} \mathrm{~B}_{4}\right) \times 100 / \mathrm{Nbre}$ de répondants.

Dans l'ensemble ces indices sont peu élevés, on note :

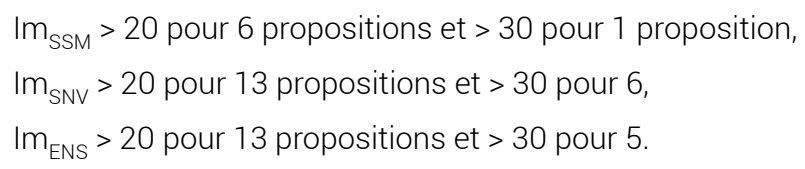

Parmi les propositions qui présentent le plus fort indice de mécontentement, 6 sont communes aux deux groupes d'étudiants ce sont :

$\begin{array}{lccc} & \text { SSM } & \text { SNV } & \text { ENS } \\ \text { P20 (Contact technologies de pointe) (T) } & 43 & 45 & 22 \\ \text { P25 (Idée travail chercheurs) (AS) } & 26 & 32 & 22 \\ \text { P26 (Temps nécessaire) (AG-AS) } & 23 & 30 & 22 \\ \text { P3 (Faire un compte rendu) (T) } & 23 & 28 & 22 \\ \text { P11 (Relations avec enseignants) (AG) } & 21 & 29 & 0 \\ \text { P22 (Augmenter intérêt physique) (AG) } & 21 & 27 & 22\end{array}$

Pour les enseignants le plus fort mécontentement se porte sur :

$\begin{array}{lrrr}\text { P12 (Esprit critique) (AS) } & 44 & 18 & 32 \\ \text { P5 (Physique et phénomènes concrets) (I) } & 33 & 14 & 20 \\ \text { P8 (Concevoir et réaliser une expérience) (T) } & 33 & 18 & 30 \\ \text { P15 (Observer et recueillir informations) (T) } & 33 & 7 & 19 \\ \text { P17 (Déterminer précision) (AS) } & 33 & 11 & 18 \\ \text { P24 (Attitude active) (AG) } & 33 & 18 & 19\end{array}$

Alors qu'ils le désirent fortement, la moitié des étudiants des deux groupes regrette de ne pas être mis en contact avec des technologies de pointe, un tiers environ de ne pas être introduit dans des laboratoires de recherche ; les enseignants, plus réalistes, conscients des difficultés que poseraient ces activités, obtiennent un Im égale à 22 seulement. Pour un quart de SSM et un tiers de SNV un mécontentement se manifeste au niveau du temps dont disposent les étudiants pour réaliser les T.P. et de la façon dont on leur apprend à faire un compte rendu. Un cinquième de SSM et un quart de SNV regrettent que les T.P. ne permettent pas d'augmenter leur intérêt pour la physique. 


\section{Conclusion}

Le résultat le plus important et qui nous a paru assez surprenant, est l'obtention d'un indice de satisfaction élevé chez l'ensemble des usagers des T.P. (enseignants et étudiants), à un degré légèrement moindre chez les étudiants de SNV. Bien évidemment, les attentes généralement importantes ne sont pas toujours comblées par la réalité. Les étudiants, surtout ceux de SNV, sont très demandeurs et de tout; les enseignants sont plus sélectifs: les propositions de type scolaire, en particulier celles relevant de l'illustration du cours ou celles conduisant à une trop grande directivité sont rejetées par un grand nombre d'enseignants. Inversement les enseignants souhaitent que les T.P. développent des attitudes scientifiques ainsi qu'une dimension créatrice et critique chez les étudiants alors que ces derniers ne le désirent pas vraiment. Les aspects qui déçoivent le plus les étudiants sont ceux qui sont en relation avec la recherche et les technologies de pointe : l'attente est forte, la réalité ne suit évidemment pas.

Au niveau de la réalité, il difficile pour les enseignants de ne pas trouver dans les T.P. ce qu'ils souhaitent tous, comme l'appréciation des ordres de grandeur, l'interprétation des résultats, le développement de l'esprit critique ; les étudiants, quant à eux, sont beaucoup plus réservés sur la réalisation de ces objectifs caractérisant des attitudes scientifiques. Ils perçoivent les T.P. sous un aspect lié aux techniques manipulatoires et à l'illustration du cours. Ils regrettent de ne pas disposer de suffisamment de temps pour les manipulations et déplorent un manque d'initiation à la rédaction d'un compte rendu. Enfin, nous avons constaté une différence entre les deux groupes d'étudiants : ceux de SSM tirent un bilan très positif, ceux de SNV sont moins satisfaits (probablement parce qu'ils sont plus demandeurs); ces derniers paraissent plus proches de la réalité mais surtout ils regrettent un manque d'ancrage scolaire et de sécurité. 


\section{Annexe 2}

\section{REALITE}

\begin{tabular}{l|l|l|}
\hline SSM & SNV & ENS \\
P2 (T) $95 \%$ & P2 (T) 85\% & P13 (T) 86\% \\
Utiliser appareils & Utiliser appareils & Présenter résultats \\
P6 (I) $81 \%$ & P10 (T) 72\% & P2 (T) 79\% \\
Vérifier théorie & Suivre instructions & Utiliser appareils \\
P23 (T) 77\% & P23 (T) 70\% & P1 (I) 71\% \\
Méthodes générales expériences & Méthodes générales expériences & Comprendre concepts \\
P5 (I) 74\% & P13 (T) 70\% & P4 (AS) 71\% \\
Physique des phénomènes concrets & Présenter résultats & Interpréter résultats \\
P15 (T) 73\% & P6 (I) 66\% & P23 (T) 71\% \\
Observer et recueillir informations & Vérifier théorie & Méthodes générales \\
& & expériences \\
P4 (AS) 70\% & P24 (AG) 66\% & P6 (I) 64\% \\
Interpréter résultats & Attitude active & Vérifier théorie \\
P8 (T) 70\% & P15 (T) 63\% & P7 (AS) 64\% \\
Concevoir et réaliser expériences & Observer et recueillir informations & Apprécier ordres de \\
& & grandeur \\
P13 (T) 68\% & P12 (AS) 64\% \\
Présenter résultats & P2\% & Esprit critique \\
P16 (I) 67\% & P9 (AG) 62\% \\
Enseigner à partir expériences & & Travailler en équipe \\
P14 (I) 66\% & & \\
Appliquer résultats & & \\
P1 (I) 66\% & & \\
Comprendre concepts & & \\
\hline
\end{tabular}




\section{SOUHAITS}

\begin{tabular}{|c|c|c|}
\hline $\begin{array}{l}\text { SSM } \\
\text { P1 (I) 92\% } \\
\text { Comprendre concepts } \\
\text { P4 (AS) } 91 \% \\
\text { Interpréter résultats } \\
\text { P8 (T) } 90 \% \\
\text { Concevoir et réaliser expérience } \\
\text { P15 (T) 90\% } \\
\text { Observer et recueillir informations } \\
\text { P20 (T) } 90 \% \\
\text { Contact technologies de pointe } \\
\text { P22 (AG) } 90 \% \\
\text { Augmenter intérêt physique }\end{array}$ & $\begin{array}{l}\text { SNV } \\
\text { P2 (T) 94\% } \\
\text { Utiliser appareils } \\
\text { P4 (AS) 94\% } \\
\text { Interpréter résultats } \\
\text { P23 (T) 94\% } \\
\text { Méthodes générales expériences } \\
\text { P24 (AG) 94\% } \\
\text { Attitude active } \\
\text { P25 (AS) 94\% } \\
\text { Idée travail chercheurs } \\
\text { P1 (I) 92\% } \\
\text { Comprendre concepts } \\
\text { P5 (I) 90\% } \\
\text { Physique et phénomènes concrets } \\
\text { P8 (T) 90\% } \\
\text { Concevoir et réaliser expérience } \\
\text { P13 (T) } 90 \% \\
\text { Présenter résultats }\end{array}$ & $\begin{array}{l}\text { ENS } \\
\text { P2 (T) 100\% } \\
\text { Utiliser appareils } \\
\text { P4 (AS) } 100 \% \\
\text { Interpréter résultats } \\
\text { P7 (AS) } 100 \% \\
\text { Apprécier ordres de } \\
\text { grandeur } \\
\text { P12 (AS) 100\% } \\
\text { Esprit critique } \\
\text { P13 (T) 100\% } \\
\text { Présenter résultats } \\
\text { P17 (T) 100\% } \\
\text { Déterminer précision } \\
\text { P23 (T) 100\% } \\
\text { Méthodes générales } \\
\text { expériences } \\
\text { P24 (AG) 100\% } \\
\text { Attitude active } \\
\text { P3 (T) 93\% } \\
\text { Faire compte rendu } \\
\text { P15 (T) 93\% } \\
\text { Observer et recueillir } \\
\text { informations }\end{array}$ \\
\hline
\end{tabular}

\section{BIBLIOGRAPHIE}

\section{Références}

BEN-ZVI R., HOSTEIN A. et SAMUEL D. : Use of an Objectives Questionnaires as a Method for Curriculum Evaluation. Science Education, 61, 3, 415-422, 1977.

BRASEIL H : The effect of Real-time Laboratory Graphing on Learning Graphic Representations of Distance and Velocity. Journal of Research in Science Teaching, 24, 4, 385-395, 1987.

CHEVALLARD Y. : La transposition didactique. Grenoble : La Pensée Sauvage, 1985.

COULTER J.C. : The Effectiveness of Inductive Laboratory, Inductive Demonstration and Deductive laboratory in Biology. Journal of Research in Science Teaching, 4, 185-186, 1966.

DOROTHY L.G., SAMUEL K.V., STANLEY H., NOVAK J. et BUTZOW J. : Research Interests of Secondary Science Teachers, Journal of Research in Science Teaching, 23, 2,145-163, 1986.

DUREY A., LAURENT M. et JOURNEAUX R. ; Confrontation Expérience-Théorie grâce à l'Ordinateur. Exemple des fentes d'Young. European Journal of Physics, 4, 2, 82-89,1983. 
DUREY A. et JOURNEAUX R. : Expérience d'Elihu Thompson, un Exemple de Modélisation par Etapes. European Journal of Physics, 8,1, 18-23, 1987.

FIRPO J.L., NEEL P. et BOYER L. : Manipulations assistées par ordinateur. XI èmes Journées Internationales sur L'Education Scientifique, Chamonix. Paris : Université Paris 7, 1989.

GHIGLIONE R. et MATALON B. : Les Enquêtes Sociologiques. Paris : Armand Colin, 1978.

GLASSON, G.E., The Effects of Hands-on and Teacher Demonstration Laboratory Methods on Science Achievement in Relation to Reasonning Ability and Prior Knowledge. Journal of Research in Science Teaching, 26, 2, 121-131, 1989.

GUTIERREZ F. A. et RODRIGUEZ L.M. : El Apprendizaje de la Fisica como Investigacion. Un ejemplo de Applicacion en la Ensenza Media. Ensenza de las Ciencias, 5, 2, 135-144, 1987.

HERRERA F. et CORULLON A. : Experiencia de Laboratoria no Estructurada en la Universidad. Ensenza de las Ciencias, 5, 2, 145-148, 1987.

HERRON M.D. : The nature of scientific enquiry. School Review, 79, 1971.

FIRPO J.L. ; NEEL P. et BOYER L. : Manipulations assistées par ordinateur. XI èmes Journées Internationales sur L'Education Scientifique, Chamonix. Paris : Université Paris 7, 1989.

JOHSUA M.A. et JOHSUA S. : Les fonctions didactiques de l'expérimental dans l'enseignement scientifique (première partie). Recherches en Didactique des mathématiques, 8, 3, 231-266, 1987.

JOHSUA M.A. et JOHSUA S. : Les fonctions didactiques de l'expérimental dans l'enseignement scientifique (deuxième partie). Recherches en Didactique des mathématiques, 9, 1, 5-30, 1988.

JOHSUA S. et DUPIN J.J : Représentations et modélisations : le « débat scientifique » dans la classe et l'apprentissage de la physique. Berne : Peter Lang, 1989.

MOKROS J.R. : The Impact of Microcomputer-Based Labs on Children Ability to Interpret Graphs. Journal of Research in Science Teaching, 24, 4, 369-383, 1987.

NACHMIAS R et LINN M.C. : Evaluations of Science Laboratory Data : the Role of Computer. Presented Information. Journal of Research in Science Teaching, 24, 5, 491-506, 1987.

PEREZ M.C. et RUBIO F. : Evaluacion de una Experiencia didactica en la Linea del Discubrimento dirigida para la Ensenza de la Fisica en $2^{\circ}$ de BUP. Ensenza de las Ciencias, 4, 3, 223-232, 1986.

READ G.A. : Experimental Evidence for Newton's Laws. European Journal of Physics, 8, 1, 6-9, 1987.

ROSELL A.C., VALERO J.S. et LLOPIS J.B.S. : Un Ejemplo de Profundizacion en los Trabajos Practicos de Fifsica : en Torno a la Caïda Libre en el Aire. Ensenza de las Ciencias, 1, 1, 38-41, 1983.

SEBASTIA J.M. : Que se Pretende en los Laboratorios de Fisica Universitaria ? Ensenza de las Ciencias, 5, 3, 196-204, 1987.

SOLOMON J. : A Study of Behaviour in the Teaching Laboratory. International Journal of Science Education, 11, 3, 317-326, 1989.

YAGER R.E., ENGLEN H.B. et SNIDER B.C. : Effects of the laboratory and demonstration methods upon the outcomes of instruction in secondary biology. Journal of Research in Science Teaching, 6,1969 .

\section{ANNEXES}




\section{Annexe 1}

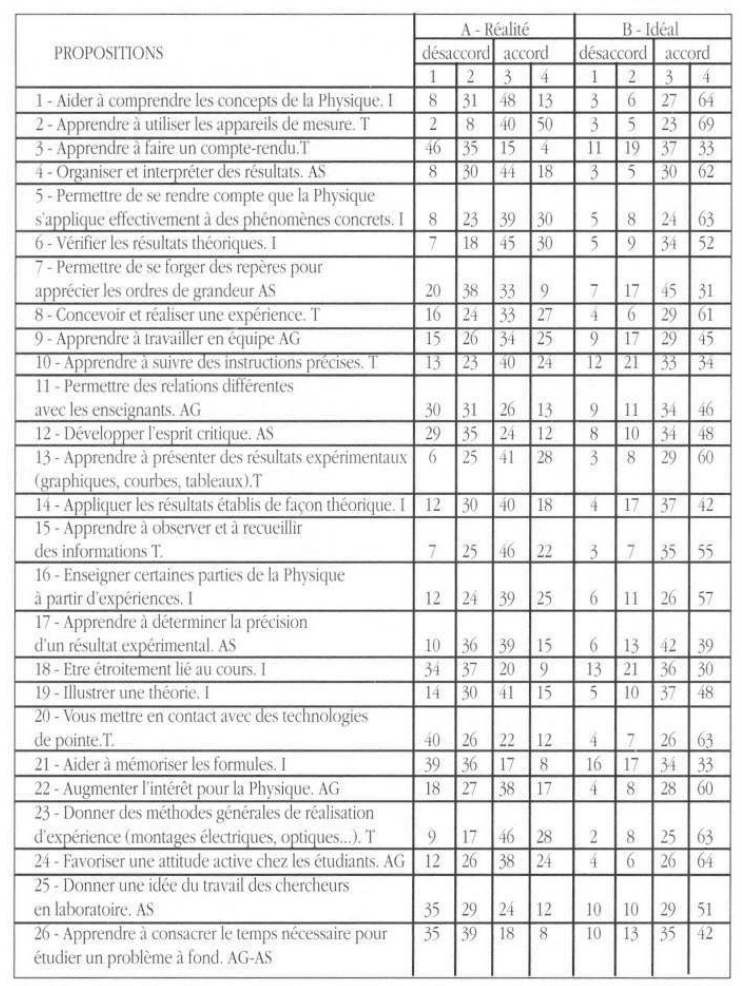

\section{RÉSUMÉS}

Le rapport à l'expérimental dans l'enseignement des sciences, et singulièrement de la Physique, est classiquement paré de toutes les vertus. Beaucoup s'accordent sur l'énoncé suivant: les difficultés de l'enseignement de la Physique proviennent de ce qu'il n'est pas basé sur des manipulations pratiques. Par ailleurs, on diagnostique souvent un échec général de l'enseignement expérimental. Pour l'essentiel, celui-ci serait surtout orienté vers les aspects purement conceptuels et fort éloigné d'une véritable activité scientifique. Dans cet article, nous cherchons à éclaircir comment les fonctions des T.P. apparaissent, subjectivement, aux acteurs : étudiants et professeurs.

The experimental attitude in the teaching of science, and of physics in particular, is classically attributed with all possible virtues. Many would agree with the following proposition: the difficulties of physics teaching come from the fact that it is not based on practical manipulations. Further more, one often hears of a general failure in the teaching of the experimental method. In this paper, we have tried to clarify how the functions of laboratory sessions appear, subjectively, to the actors : students and instructors.

\section{INDEX}

Mots-clés : catégorie d'objectif, indice de mécontentement, indice de satisfaction, outil conceptuel, technique manipulatoire 


\section{AUTEURS}

\section{GÉNIN}

Laboratoire de physique Apprentissage et Didactique, université de Provence, Marseille

\section{A. PELLET}

Laboratoire de physique Apprentissage et Didactique, université de Provence, Marseille 